\title{
HIPERPLASIA GENGIVAL INDUZIDA POR CILLOSPORINA A
}

\author{
Vera lúcia Costa Ramalho, Horácio José Ramalho, José Paulo Cipullo, Emmanuel A. Burdmann* \\ Disciplina de Nefrologia da Faculdade de Medicina de São José do Rio Preto
}

RESUMO - 0 crescente uso da ciclosporina A (CSA) em transplantes de órgãos e no tratamento de doenças auto-imunes aumentou a incidência de seus efeitos adversos, entre eles a hiperplasia gengival (HG). Esta acarreta problemas estéticos, de fala, mastigação e de erupção dentária nos pacientes afetados. A prevalência de hiperplasia gengival induzida por ciclosporina varia nos diversos estudos, podendo chegar a $85 \%$, dependendo do critério utilizado para seu diagnóstico. Esta revisão aborda aspectos etiológicos, histológicos, de quadro clínico, prevenção e tratamento desta importante lesão.

UnITERMOS: Ciclosporina A. Toxicidade. Hiperplasia Gengival.

\section{INTRODUÇÃo}

A ciclosporina tem sido usada quase que universalmente na prevenção da rejeição de transplantes de órgãos. Desde a sua utilização inicial em transplantados renais, em 1978', vem sendo empregada sozinha ou em combinação com outras drogas imunossupressoras para a prevenção da rejeição de transplantes de rim, fígado, pâncreas, medula óssea, intestino, coração e pulmão. O número de pacientes recebendo esta droga aumentou ainda mais com o seu uso no tratamento de doenças auto-imunes como artrite reumatóide, psoríase, líquem plano, pênfigo bolhoso, esclerose múltipla, lúpus eritematoso, miastenia grave, diabetes mellitus, uveites e diversas glomerulopatias ${ }^{2,3}$.

AciclosporinaA pode ser administrada por via oral, intramuscular ou intravenosa, em doses variáveis, dependendo da situação a ser tratada. A seleção da dose ótima de tratamento é usualmente baseada na mensuração individual dos níveis de ciclosporina no sangue. $A$ meta é conseguir levar a dose de ciclosporina de cada paciente ao mais baixo nível efetivo. Novas formulações de microemulsão de ciclosporina começaram a ser utilizadas recentemente. Essas fórmulas têm melhor absorção intestinal, reduzindo a acentuada biovariação observada com as soluções tradicionais de CSA em óleo de oliva ${ }^{4}$.

\footnotetext{
* Correspondência:

Faculdade de Medicina de São José do Rio Preto

Av. Brigadeiro Faria Lima 5416

São José do Rio Preto - SP

15090-000 - Telefone: (17) 210.5712

Email: burdmann@famerp.br
}

Apesar de seu inequívoco sucesso, a ciclosporina é associada a vários efeitos adversos. Muitos deles são dose dependentes e potencialmente reversíveis quando da diminuição ou descontinuação da droga. Os seus principais efeitos colaterais são nefrotoxicidade, hepatoxicidade, hipertensão, neurotoxicidade, aumento da predisposição a infecções bacterianas, fúngicas e virais, ealterações metabólicas (hiperglicemia, hipercolesterolemia $)^{5,6}$. Em termos odontológicos, o efeito colateral mais notável da CSA é o desenvolvimento de hiperplasia gengival (HG).

\section{Histórico e prevalência}

A primeira descrição de um caso de crescimento gengival causado por medicamentos data de 1939 e foi associada ao uso de anticonvulsivantes ${ }^{7}$. Atualmente, três categorias de drogas são associadas com crescimento gengival iatrogênico $(\mathrm{HG})$ : anticonvulsivantes (especialmente fenitoina), agentes bloqueadores de canais de cálcio como a nifedipina, e o imunossupressor ciclosporina ${ }^{8}$. Aocorrência deste fenômeno pode interferir com a fala, mastigação e erupção dentária e ser esteticamente muito desagradável.

Em 1983 surgiram os primeiros relatos evidenciando que a CSA provocava $\mathrm{HG}$ em receptores de transplantes de órgãos ${ }^{9}$. A prevalência de hiperplasia gengival induzida por CSA varia amplamente entre os diversos estudos existentes (13\% a 85\%), dependendo do critério utilizado ${ }^{10,11}$. A determinação da prevalência real é difícil, pois existem diversos fatores de confusão nas populações sujeitas, como diferentes doses de droga e concentração sérica de CSA, duração da terapia, méto- dos de avaliação de crescimento gengival, condição periodontal básica, idade dos pacientes, condição médica para a qual a droga está sendo usada, saúde geral do paciente e uso concomitante de outros medicamentos (especialmente aqueles associados com crescimento gengival). A prevalência de $\mathrm{HG}$ independe do fato da CSA estar sendo usada para a prevenção da rejeição de órgãos ou para tratamento de outras desordens sistêmicas ${ }^{5}$.

\section{Características clínicas}

No paciente susceptível, o crescimento gengival freqüentemente acontece entre uma três meses após o início da terapia com ciclosporina ${ }^{12}$. A extensão da hiperplasia pode variar desde leve mudança no contorno do tecido da papila gengival até a completa cobertura dos dentes, interferindo com a oclusão, mastigação e fala.

Os tecidos gengivais afetados sangram prontamente à sondagem e são geralmente mais hiperemiados que os tecidos gengivais normais, mostrando marcantes mudanças inflamatórias. Todos os segmentos da dentição podem ser afetados, mas o segmento anterior parece ser a área mais propensa ao desenvolvimento de $\mathrm{HG}^{13}$. O aumento gengival é mais pronunciado no aspecto labial da gengiva do que na face palatina ou lingual ${ }^{14}$. A hiperplasia gengival induzida por CSA não foi observada em pessoas edêntulas ${ }^{15}$.

Além dos óbvios problemas estéticos, 0 crescimento pode resultar em áreas sem higiene que são mais propensas às cáries, desenvolvimento de periodontites e infeccões que podem levar a septicemia. $O$ crescimento gengival freqüentemente induz 
a dificuldades nutricionais, especialmente em crianças, nas quais pode alterar a erupção normal dos dentes ${ }^{16}$.

\section{Características histológicas}

As características histológicas de todas as hiperplasias gengivais induzidas por drogas são semelhantes, consistindo principalmente de tecido conjuntivo com um revestimento irregular de múltiplas camadas de epitélio paraqueratinizado de espessura variável. Sulcos epiteliais penetram no tecido conjuntivo subepitelial, produzindo feixes irregulares de fibras colágenas. Este tecido conjuntivo éaltamente vascularizado e caracterizado por acúmulo de células de infiltrado inflamatório. O tipo de célula predominante no infiltrado é o macrófago, com linfócitos sendo observados em menor proporçãa ${ }^{6}$.

Alguns pesquisadores têm notado certo grau de fibroplasia dentro do tecido conjuntivo gengival, caracterizado pela presença de grande número de fibroblastos ${ }^{12}$. A maioria dos estudos, no entanto, não demonstrou aumento na densidade numérica de fibroblastos e colágeno extracelular em relação à gengiva normal ${ }^{17,18,19}$. Isto significa que o crescimento gengival induzido por ciclosporina não deve ser uma verdadeira hiperplasia. Por essa razão, o termo hiperplasia gengival tem sido substituído por crescimento gengivalem publicações mais recentes. Análises ultra-estruturais têm demonstrado que fibroblastos gengivais mostram características de síntese e secreção ativas de proteína, com reduzida citotoxicidade ou mudanças degenerativas no crescimento gengival induzido por ciclosporina. $O$ aumento de fibroblastos especializados, chamados miofibroblastos, também tem sido observado no crescimento gengival associado ao uso da $\mathrm{CSA}^{20}$.

\section{Mecanismos potenciais da lesão}

O mecanismo exato de ação pelo qual a ciclosporina age simultaneamente como um imunossupressor seletivo enquanto provoca reação tecidual indesejável continua pouco entendido. Como nem todos os pacientes desenvolvem o crescimento gengival, os termos respostae não respostatêm sido utilizados na literatura para identificar, respectivamente, a existência ou não de susceptibilidade individual e talvez predisposição genética.

A idade e o sexo do paciente podem ser fatores adicionais que influenciam a prevalência e gravidade do crescimento gengival induzido por CSA. Estudos clínicos sugerem que crianças, especialmente adolescentes e mulheres jovens, são mais susceptíveis ao crescimento gengival causado por CSA que adultos $^{14,21}$. Isso pode estar relacionado à existência de um fenótipo fibroblástico peculiar a pacientes jovens ou à influência de hormônios sexuais. Aumento de testosterona biologicamente ativa tem sido encontrado nos tecidos crescidos dos pacientes com aumento gengival induzido por droga ${ }^{22}$, sugerindo que alterações do metabolismo andrógeno podem causar propensão ao aumento de crescimento gengival induzido por CSA em crianças e adolescentes ${ }^{14}$. Por outro lado, um estudo realizado para determinar fatores de risco no desenvolvimento de HG induzida por CSA não conseguiu demonstrar associação com idade e sexo do paciente $\mathrm{e}^{23,24}$.

A relação entre prevalência e gravidade de crescimento gengival com a dose e concentração sérica de CSA é ainda assunto controverso. Alguns estudos clínicos demonstraram que a prevalência de crescimento gengival foi relacionada a altas doses de CSA ou alta concentração sangüínea da droga ${ }^{18,23,24,25}$, enquanto outros não conseguiram confirmar esta correlação ${ }^{26,27,2)}$.Tem sido postulado que um limiar de concentração definido da droga énecessário para induzir a reação gengival e que níveis de droga acima deste limiar não aumentariam a gravidade da lesão $0^{12,27}$. As concentrações de droga na saliva, fluido cérvico gengival ou placa bacteriana também podem estar relacionados à expressão e patogênese do crescimento gengival induzido pela CSA. Alguns autores têm demonstrado correlação positiva entre concentração de CSA na saliva estimulada e a extensão do crescimento gengival| ${ }^{27,28}$, enquanto outros não evidenciaram esta correlação $0^{29,30}$. Estes achados conflitantes podem estar ligados ao fato da placa dental agir como um reservatório potencial para a CSA, que seria liberada pela ação do escoamento estimulado ${ }^{14}$. Esta hipótese é suportada pelo achado de concentrações de CSA na placa dental mais elevadas que aquelas encontradas no sangue ou outros tecidos 5 .

Existem evidências de que a placa induz à inflamação gengival e exacerba o crescimento gengival induzido por $\mathrm{CSA}^{26,27,28}$. Além disso, a maioria dos estudos sugere que 0 controle efetivo da placa e remoção de irritantes locais minimizam a intensidade do crescimento gengival induzido por CSA e previnem a recorrência de lesões gengivais ${ }^{10,28,29,30,31}$. Estes dados sugerem que a inflamação gengival induzida por placa deve ser um fator importante no desenvolvimento do crescimento gengival induzido por CSA. Entretanto, não estáainda claro se a placa é fator contribuitório ou conseqüência das alterações gengivais. De fato, vários estudos bem controlados não conseguiram demonstrar correlação entre placa, inflamação gengival e crescimento induzido por CSA ${ }^{12,28}$. Adicionalmente, tem sido mostrado que controle ótimo da placa e remoção local de irritantes não preveniram o desenvolvimento do crescimento gengival por CSA $A^{28,31}$.

Em conjunto, estes dados sugerem que a inflamação gengival induzida por placa aumenta a intensidade do crescimento gengival induzido por CSA. Por esta razão seria razoável assumir que higiene oral excelente poderia diminuir a gravidade do crescimento gengival induzido por CSA, através da eliminação do componente inflamatório da lesão. No entanto, não é provável que um ótimo controle de placa previna significantemente o desenvolvimento do crescimento gengival.

Mais recentemente, tem se estudado a função das citocinas e fatores de crescimento na patogênese do crescimento gengival induzido por drogas ${ }^{32,33}$. Estas investigações são baseadas na premissa de que a homeostase celular em tecidos é, provavelmente, resultado de um balanço entre interações complexas de moléculas antagonistas, no qual citocinas e fatores de crescimento fazem a maioria das funções. Os mecanismos celulares e moleculares do crescimento gengival induzido por CSA podem estar relacionados a mudanças no fenótipo dos macrófagos. Por exemplo, demonstrou-se que a fenitoina causa aumento na expressão do RNA mensageiro (mRNA) e secreção de PDGF em cultura de macrófagos e monócitos ${ }^{32}$, sugerindo possível participação desta citocina no crescimento gengival induzido pela fenitoina. $\mathrm{Da}$ mesma forma, estudos recentes demonstraram que mRNA para PDGF-B estava significantemente aumentado em tecidos 
Ramalho VlC et al.

gengivais crescidos de pacientes tratados com CSA quando comparados a controles normais ${ }^{33}$ e que a expressão de interleucinal, interleucina 6 e fator de necrose tumoral encontra-se aumentada no fluido gengival crevicular de pacientes tratados com CSA ${ }^{34}$.

\section{Prevenção e tratamento}

O tratamento e a prevenção do crescimento gengival induzido por drogas permanecem insatisfatórios. Embora a mudança na dosagem de CSA possa ser considerada eficaz do ponto de vista odontológico, nem sempre é possível na prática médica, pela possibilidade de perda do enxerto. Assim, para $\circ$ paciente transplantado, há pouca chance de suspender ou diminuir a droga, e cirurgias gengivais repetidas continuam sendo a opção de tratamento $0^{8,35}$.

A maioria dos autores concorda que um programa de higiene oral atua positivamente sobre a hiperplasia gengival induzida por ciclosporina, diminuindo o grau de inflamação, sangramento e dor, mas mostra-se impotente na diminuição do crescimento gengival $^{8,12,28,36}$.

Recentemente, descreveu-se a utilidade do antibiótico macrolídeo azitromicina no tratamento deste tipo de hiperplasia ${ }^{37-40}$. A descrição inicial baseou-se nos resultados obtidos casualmente em dois transplantados renais que após tratamento de infecção respiratória com azitromicina apresentaram significativa redução de $\mathrm{HG}^{38}$. A dose utilizada tem sido de $250 \mathrm{mg}$ a $500 \mathrm{mg}$ em três a cinco dias de tratamento. Este efeito parece ser específico da azitromicina, pois antibioticoterapia com metronidazol produziu resultados conflitantes em relação à redução da $\mathrm{HG}^{41,42}$.

Publicações recentes sugerem que a substituição da ciclosporina por tacrolimus pode reduzir significativamente a $\mathrm{HG}^{43,44,45}$.

\section{SUMMARY \\ Ciclosporine A-Induced gingival HYPERPLASIA}

The increasing use of cyclosporine $A(C S A)$ in organ transplants and in the treatment of autoimmune diseases has increased the incidence of cyclosporine A-related adverse effects, including gingival hyperplasia (GH). GH causes esthetic, speech, mastication and tooth growth problems in the affected patients. The prevalence of cyclosporine-induced $G H$ varies in different studies and may be as high as $85 \%$, depending on the diagnostic criteria. This review approaches etiological, histological and clinical issues, as well as the prevention and treatment of this important injury. [Rev Assoc Med Bras 2003; 49(2): 210-3]

KEY wORDs: Cyclosporine-A. Toxicity. Gingival hyperplasia.

\section{REFERÊNCIAS}

I. Calne RY, Thiru S, McMaster P, Cradock GN, White DJG, Evans DB, et al. Cyclosporine A in patients receiving renal allografts from cadaver donors. Lancet 1978; I: I323-7.

2. Kahan BD. Cyclosporine. NEngl J Med I989; 321: 1725-38.

3. de Mattos AM, Olyaei AJ, Bennett WM. Pharmacology of immunosuppressive medications used in renal diseases and transplantation. Am J Kidney Dis 1996; 28:63 I-67.

4. Johnston A, Holt DW. Cyclosporine: What should we be looking for in newformulations? Transplant Proc 1998; 30: 1652-3.

5. Marshall RI, Bartold MD. A clinical review of drug - induced gingival overgrowth. Aust Dent J 1999; 44:219-32.

6. Boltchi FE, Rees Terry D, lacopino AM. Cyclosporine A induced gingival overgrowth: A comprehensive review. Quintessence Int 1999; 30:775-83.

7. Kimball OP. The treatment of epilepsy with sodium diphenyl-hydantoinate. JAMA 1939; I 12:1244-5.

8. Stephen J Meraw, DDS, Phillip J Sheridan, DDS. Medically induced gingival hyperplasia. Mayo Clin Proc 1998; 73: I 196-99.

9. Rateitschak-Pluss EM, Hefti A, Loertscher R, Thiel G. Initial observation that cyclosporine-A induces gingival enlargement in man. J Clin Periodontol I 983; 10:237-46.

10. Wondimu B, Dahllof G, Berg U, Modeer T. Cyclosporine-A induced gingival overgrowth in renal transplant children. Scand J Dent Res 1993; 101:282-6.

II. Allman SD, McWhorter AG, Seale NS. Evalution of cyclosporine induced gingival overgrowth in the pediatric transplant patient. Pediatr Dent 1994; 16:36-40.

12. Seymour RA, Smith DG, Rogers SR. The comparative effects of azathioprine and cyclosporine on some gingival health parameters of renal transplant patients. J Clin Periodontol 1987; 14: 610-3.

13. Tyldesley W, Rotter E. Gingival hyperplasia induced by cyclosporin-A. Br Dent J 1984; 157:305-9.

14. Seymour RA, Thomason JM, Ellis JS. The pathogenesis of drug-induced gingival overgrowth. J Clin Periodontol I 996; 23: 165-75.

15. FriskoppJ, Klintmalm G. Gingival enlargement: a comparison between cyclosporine and azathioprine treated renal allograft recipients. Swed Dent J 1986; 10: 85-92.

16. KilpatrickNM, Weintraub RG, LucasJO, Shipp
A, Byrt T, Wilkinson JL. Gingival overgrowth in pediatric heart and hearth-lung transplant recipients. J Heart Lung Transplant 1997; 16:1231-7.

17. Wisocki GP, Gretzinger HA, Laupacis A, Ulan RA, Stiller CR. Fibrous hyperplasia of gingiva: $A$ side effect of cyclosporine- A therapy. Oral Surg Oral Med Oral Pathol 1 983; 55:274-8.

18. Rostock MH, Fry HR, Turner JE. Severe gingival overgrowth associated with cyclosporine therapy.J Periodontol 1986; 57:294-9.

19. Bonnaure-Mallet M, Tricot-Doleux S, Godeau GI. Changes in extracellular matrix macromolecules in human gingiva after treatment with drugs inducing gingival overgrowth. Arch Oral Biol I 995; 40:393-400.

20. Dill RE, lacopino AM. Myofibroblastos in phenitoin-induced hyperplastic connective tissue in the rat and in human gingival overgrowth. J Periodontol 1997; 68:375-80.

2I. Pernu HE, Knuuttila MLE, Huttunen KRH, Tiilikainen ASK. Drug-induced gingival overgrowth and class II major histocompatibility antigens. Transplantation 1994; 57: I8II-3.

22. Sooriyamoorthy M, Gower DB, Eley BM. Androgen metabolism in gingival hyperplasia induced by nifedipine and cyclosporine. J Periodontol Res 1990; 25:25-30.

23. Thomas DW, Newcombe RG, Osborne Gr. Risk factors in the development of cyclosporine-induced gingival overgrowth. Transplantation 2000; 69:522-6.

24. Thomas DW, Baboolal K, Subramanian N, Newcombe RG. Cyclosporin A-induced gingival overgrowth is unrelated to allograft function in renal transplant recipients. J Clin Periodontol 200 I; 28:706-9.

25. Adams D, Davies G. Gingival hyperplasia associated with cyclosporine $A$ : a report of 2 cases. Br Dent J 1984; 157: 89-90.

26. McGaw WT, Lam S, Coates J. Cyclosporineinduced gingival overgrowth: correlation with dental plaque scores, gingivitis scores, and cyclosporine levels in serum and saliva. Oral Surg Oral Med Oral Pathol 1987; 64: 293-7.

27. Daley TD, Wysocki GP, Day C. Clinical and pharmacological correlations in cyclosporineinduced gingival hyperplasia. Oral Surg Oral Med Oral Pathol 1986; 62: 7-21.

28. Seymour RA, Smith DG. The effect of a plaque control program on the incidence and severity of cyclosporine- induced gingival changes. J Clin Periodontol I99I; I 8: 107-I0.

29. King GN, Fullinfaw R, Higgins TS, Walker RJ, Francis DMA, Wiesenfeld D. Gingival hyperplasia in renal allograft recipients receiving cyclosporine $A$ and calcium antagonists. J Clin Periodontol 1993; 20:286-93.

30. Pernu HE, Pernu LMH, Huttunen KRH, Niemen PA, Knuuttila MLE. Gingival overgrowth among renal transplant recipients related to immunosuppressive medication and possible local background factors. J Periodontol 1992; 63:548-53.

31. Somacarrera ML, Hernandez G, Acero J, Moskow BS. Factors related to the incidence 
and severity of cyclosporine-induced gingival overgrowth in transplant patients: A longitudinal study. J Periodontol 1994; 65:67I-5.

32. Dill RE, Millre EK, Weil T, Lesley S, lacopino AM. Phenytoin increases gene expression for platelets-derived growth factor B chain in macrophages and monocytes. J Periodontol 1993; 64:169-73.

33. Nares S, Ng MC, Dill RE, Park B, Cutler CW, lacopino AM. Cyclosporine-A upregulates platelet-derived growth factor B chain in hyperplastic human gingiva. J Periodontol 1996; 67:27I-8.

34. Atilla G., Kutukculer N. Crevicular fluid interleukin-I beta, tumor necrosis factor-alpha, and interleukin-6 levels in renal transplant patients receiving cyclosporine A. J Periodontol 1998; 69:784-90.

35. Darbar UR, Hopper C, Speight PM, Newman $\mathrm{HN}$. Combined treatment approach to gingival overgrowth due to drug therapy. J Clin Periodontol 1996; 23:94 I-4.

36. McGaw T, LamS, Coates J. Cyclosporin induced gingival overgrowth: correlation with dental plaque scores, gingivitis scores and cyclosporin levels in serum and saliva. Oral Surg Oral
Med Oral Pathol 1987; 64:293-7.

37. Wahlstrom E, Zamora JU, Teichman S. Improvement in cyclosporine associated gingival hiperplasia with azithromycin therapy. N Engl J Med 1995; 332:753.

38. Nash MN, Zaltzman JS. Efficacy of azithromycin in the treatment of cyclosporineinduced gingival hyperplasia in renal transplant recipients. Transplantation 1998;65:16 I I-5.

39. E. Gómez, M. Sánchez-Nuñes, J. E. Sánchez, C. Corte, S. Aguado, C. Portal, J. Baltar and J. Alvarez-Grande. Treatment of cyclosporin-induced gingival hyperplasia with azithromycin. Nephrol Dial Transplant 1997; 12:2694-7.

40. Wirnsberger GH, Pfragner R, Mauric A, Zach $\mathrm{R}$, Bogiatzis A, Holzer H. Effect of antibiotic treatment with azithromycin on cyclosporine A-induced gingival hyperplasia among renal transplant recipients. Transplant Proc 1998; 30:2117-9.

4I. Wong W, Hodge MG, Lewis A, Sharpstone P, Kingswood JC. Resolution of cyclosporininduced gingival hypertrophy with metronidazole. Lancet 1994; 343:986.

42. Aufricht C, Hogan EL, Ettenger RB. Oral metronidazole does not improve cyclosporine A-induced gingival hyperplasia. Pediatr Nephrol 1997; I I:552-5.

43. Busque S, Demers P, Saint-Louis G, Boily JG, Tousignant J, Lemieux F, Martin G, Smeesters C, Corman J, Daloze P.Hypertrichosis and gingival hypertrophy regression in renal transplants following the substitution of cyclosporin by tacrolimus. Ann Chir 1999; 53:687-9.

44. Kohnle M, Lutkes P, Zimmermann U, Philipp $T$, Heemann U. Conversion from cyclosporine to tacrolimus in renal transplant recipients with gum hyperplasia. Transplant Proc 1999; 3I:44S-5S.

45. Harikrishnan P, Harden PN. Tacrolimus can resolve cyclosporin-induced gingival hyperplasia. Nephrol Dial Transplant 1999; 14:1805-6.

Artigo recebido: 29/10/2002 Aceito para publicação: | |4/ I I/2002 